\title{
INTERVENÇÕES ASSISTIDAS POR ANIMAIS REALIZADAS EM AMBIENTE HOS- PITALAR NA PROMOÇÃO DO CUIDADO COM A VIDA
}

\author{
ASSISTED INTERVENTIONS BY ANIMALS CARRIED OUT IN A HOSPITAL ENVIRONMENT INT
}

THE PROMOTION OF LIFE CARE

Camila Moura de Lima ${ }^{1}$ Fernanda Dagmar Martins Krug² Daniele Dornelles Bender ${ }^{3}$

Mara Regina Marques Rodrigues ${ }^{4}$ Beatriz Maksud Mechereffe ${ }^{5}$

Ana Cláudia Garcia Vieira ${ }^{6}$ Sabrina De Oliveira Capella ${ }^{7}$ Márcia de Oliveira Nobre ${ }^{8}$

\section{RESUMO}

A Intervenção Assistida por Animais é um método que utiliza os animais como mediadores de um processo terapêutico. Dessa maneira, essa prática pode ser utilizada em diversos públicos de pacientes como estratégia na promoção do cuidado com a vida. Diante dos diversos benefícios, que essa interação pode proporcionar aos seres humanos, o objetivo deste estudo foi verificar os benefícios das Intervenções Assistida por Animais, realizada em pacientes hospitalares e psiquiátricos na cidade de Pelotas. Assim, as atividades realizadas ocorreram semanalmente, por um período de um ano. E a cada intervenção participavam em média cinco assistidos e também três cães co-terapeutas. Nesse contexto, foram evidenciados inúmeros benefícios para as crianças hospitalizadas, seus acompanhantes e também para os pacientes psiquiátricos. A presença dos cães ameni-

\footnotetext{
${ }^{1}$ Camila Moura de Lima - UFPEL - Brasil - Graduada em Medicina Veterinária pela Universidade Federal de Pelotas, residente multiprofissional na área de Pet Terapia pela Universidade Federal de Pelotas, camila.moura. lima@hotmail.com; ${ }^{2}$ Fernanda Dagmar Martins Krug - UFPEL - Brasil - Graduada em Medicina Veterinária pela Universidade de Cruz Alta, mestre em Ciências pela Universidade Federal de Pelotas, fernandadmkrug@gmail. com; ${ }^{3}$ Daniele Dornelles Bender - UFPEL - Brasil - Graduada em Terapia Ocupacional pela Universidade Federal de Pelotas, mestranda no Programa de Pós-graduação em Educação pela Universidade Federal de Pelotas, dornellesdaniiele@gmail.com; ${ }^{4}$ Mara Regina Marques Rodrigues - UFPEL - Brasil - Graduada em Terapia Ocupacional pela Universidade Federal de Pelotas, mara.rmr@hotmail.com; ${ }^{5}$ Beatriz Maksud Mechereffe - UFPEL Brasil - Graduada em Psicologia pela Universidade Católica de Pelotas, especialista em saúde mental e atenção psicossocial pelo Instituto Superior de Ensino, Pesquisas e Extensão, biamak@gmail.com; ${ }^{6}$ - Ana Cláudia Garcia Vieira - UFPEL - Brasil - Graduada em Enfermagem pela Universidade Federal do Rio Grande do Sul, Doutora em saúde da criança pela Universidade Católica do Rio Grande do Sul, cadicha10@gmail.com; ${ }^{7}$ Sabrina De Oliveira Capella - UFPEL - Brasil - Graduada em Medicina Veterinária pela Universidade Federal de Pelotas, mestre em Ciências pela Universidade de Pelotas, capelas.oliveira@gmail.com; ${ }^{8}$ Márcia de Oliveira Nobre - UFPEL - Brasil Graduada em Medicina Veterinária pela Universidade Federal de Pelotas, doutora em Ciências pela Universidade Federal do Rio Grande do Sul, coordenadora do projeto Pet Terapia da Universidade Federal de Pelotas, marciaonobre@gmail.com
} 
zou a saudade de casa, despertando sentimentos de empatia, afeição e melhora de humor. Conclui-se que, as intervenções assistidas por animais realizadas em pacientes hospitalares em ambas instituições da cidade de Pelotas, foram realmente benéficas. Pois, foram constatados pelos profissionais envolvidos inúmeros benefícios, como a interação social, mudança de humor, melhora na motricidade e na qualidade de vida.

Palavras-chaves: Pet terapia. Assistidos. Cães.

\begin{abstract}
Assisted Animal Intervention is a method that uses animals as mediators of a therapeutic process. In this way, this practice can be used in several patient groups as a strategy to promote care with life. In view of the many benefits that this interaction can offer to humans, the objective of this study was to verify the benefits of Animal-Assisted Interventions performed in hospital and psychiatric patients in the city of Pelotas. Thus, the activities carried out occurred weekly, for a period of one year. Each intervention involved an average of five assisted and three dogs therapists. In this context, numerous benefits were evidenced for the hospitalized children, their companions and also for the psychiatric patients. The presence of the dogs added to the homesickness, arousing feelings of empathy, affection and improvement of mood. It was concluded that the interventions assisted by animals performed in hospital patients in both institutions of the city of Pelotas, were beneficial. For the professionals involved found numerous benefits, such as social interaction, mood swings, improved motor skills and quality of life.
\end{abstract}

Keywords: Pet therapy. Assisted. Dogs.

\title{
INTRODUÇÃO
}

A qualidade de vida, de um modo geral, envolve alterações internas e externas ao indivíduo (SILVA, 2005). Dessa forma, um achado relevante encontrado em ambiente hospitalar é a alteração de humor dos pacientes internados podendo corresponder de $20 \%$ a $50 \%$ dos casos(MARCOLINO, 2007). Em vista disso, é de grande importância buscar estratégias que promovam um cuidado mais humanizado e que possam favorecer o bem-estar dos pacientes (SILVA, 2005). Diante de novos métodos, que podem ser inseridos nesse contexto, surge a utilização de animais como mediadores de um processo terapêutico. Para a realização dessa prática necessita-se de uma equipe multiprofissional, podendo envolver terapeutas ocupacionais, enfermeiros, médicos, psicólogos, profissionais da educação e médicos veterinários (NOBRE, 2017). 
As Intervenções Assistidas por Animais (IAAs) promovem uma melhora na comunicação, socialização, cognição e na qualidade de vida dos pacientes envolvidos, podendo ser subdividas em três categorias como: Atividade Assistida por Animais (AAA) - caracteriza-se por atividades que envolvem recreação, lazer e entretenimento dos assistidos; Terapia Assistida por Animais (TAA) - necessita de um planejamento prévio das atividades, o envolvimento de um profissional da saúde, possui um objetivo específico e Educação Assistida por Animais (EAA) - são atividades que buscam a melhora no processo ensino-aprendizagem e há o envolvimento de um profissional da educação. Essas práticas estão sendo inseridas em algumas instituições de saúde e sendo desenvolvidas com diversos tipos de pacientes como, por exemplo, crianças hospitalizadas e pacientes psiquiátri$\cos$ (ABRHÃO, 2015; CHELINI; OTTA, 2016).

Estudos relatam os efeitos, que as IAAs podem proporcionar aos seres humanos, dentre eles, uma melhora na comunicação, socialização, autoestima, estabelecimento de vínculos afetivos, sensação de bem-estar, diminuição da ansiedade, agressividade, hiperatividade e da sensação da dor em crianças hospitalizadas. O contato com os animais auxilia também na redução dos níveis da pressão arterial, colesterol, estresse e melhora na saúde cardiovascular (GODOY, 2007; RIBEIRO, 2011; ICHITANI, 2015).

Deste modo, o objetivo deste estudo foi verificar os benefícios das Intervenções Assistida por Animais, realizada em pacientes hospitalares e psiquiátricos na cidade de Pelotas.

\section{METODOLOGIA}

O projeto Pet Terapia, da Universidade Federal de Pelotas, desenvolve Intervenções Assistida por Animais desde o ano de 2006 na cidade de Pelotas e região, e é situado no campus Capão do Leão/UFPel. É composto por uma equipe multiprofissional de docentes e discentes da graduação e pós-graduação. Para atuar como co-terapeutas os cães passam por um controle rígido de saúde, sendo necessárias avaliações periódicas através de exames complementares. Os cães são capacitados e treinados a se tornarem aptos a desenvolver as atividades propostas durante as sessões de IAAs.

As sessões de Intervenções Assistidas por Animais foram desenvolvidas em duas instituições hospitalares da cidade de Pelotas/RS, os encontros foram realizados uma vez na semana, com duração aproximada de 40 minutos, por um período deum ano. A equipe participante das IAAs caracterizou-se por envolver profissionais e acadêmicos de diversas áreas como enfermagem, terapia ocupacional, psicologia, medicina veterinária. As atividades foram desenvolvidas em 
local dentro das instituições pré-destinadaspara as sessões de IAA. A cada intervenção participava em média cinco pacientes e também três cães co-terapeutas.

Durante as IAAs buscou-se estabelecer um vínculo entre o paciente e o animal, onde esse vínculo é estabelecido na fase inicial das atividades através da aproximação e da relação com os cães co-terapeutas. Na fase intermediária da intervenção buscou-se desenvolver atividades específicas como, a simulação de procedimentos hospitalares nos cães, realização de atividades lúdicas com a temática dos animais e também a realização de caminhadas. Na fase final trabalhou-se a despedida dos animais, através de jogos interativos. Dessa forma, procurou-se envolver atividades que promovessem uma melhora na afetividade, interação social, cognição e a motricidade dos assistidos envolvidos.

\section{RESULTADOS E DISCUSSÃO}

Durante as intervenções foi possível observar inúmeros benefícios aos assistidos, acompanhantes e a equipe envolvida. Nas crianças hospitalizadas foi possível observar de acordo com o relato dos profissionais envolvidos, que durante as atividades as crianças sentiram-se bem com a presença dos cães, houve uma amenização da saudade de casa, despertou sentimentos de afetividade e melhora no humor. Também foi possível observar que antes das intervenções, as crianças e os acompanhantes encontravam-se abatidos e tristes, e após o término das atividades, houve uma mudança de humor e descontração de acordo com os profissionais envolvidos.

Esses resultados corroboram com pesquisas recentes, que reconhecem os efeitos benéficos que as intervenções assistidas por animais proporcionam a crianças hospitalizadas (VACCARI, 2017), como melhora na autoestima, distração, entretenimento e mudança de humor. Além disso, tornam o ambiente hospitalar mais humanizados, favorecendo uma interação entre crianças, familiares e equipe (KOBAYASHI et al., 2009).

Outros benefícios evidenciados pelos profissionais envolvidos nas sessões de IAA, foi que durante o período da internação, as crianças apresentaram maior mobilidade fora do leito, pois apresentavam-se receptivas para participar das atividades propostas. Mas, durante as visitas dos cães, foi perceptível uma maior atividade física das mesmas. Também houve melhora na comunicação, amenização da dor, desconforto, fome, sede, diferentemente do que ocorre quando estão desmotivadas. Nesse contexto, podemos destacar que a presença dos cães, auxiliou na redução da ansiedade, estresse, depressão e solidão, estimulando a atividade física e consequentemente a elevação do bem-estar dos assistidos (SILVEIRA, 2011). 
Foi relatado ainda, que as crianças apresentaram uma maior receptividade ao receber os cuidados, pois houve uma amenização do medo durante a realização de procedimentos como, por exemplo, a administração de medicações, punções, exames e cirurgias. E também após as atividades, quando retornavam aos leitos, os pacientes expressavam sentimentos de euforia e felicidade. $E$ as atividades propostas posteriormente às intervenções com os cães, eram conduzidas com mais disposição e através delas podia-se acessar informações relacionadas a sentimentos, dores e etc. Em razão disso, muitas vezes os pacientes relembraram dos seus animais de estimação e comparam com os cães coterapeutas. Assim, foi possível promover o estímulo da memória dos assistidos e ajudar a relembrar memórias do passado (YAMAMOTO, 2012).

Já nas IAAs realizadas com pacientes psiquiátricos adultos, acometidos por depressão, foi possível constatar melhora emocional, demonstrada através da empatia e afeiçãopelos cães participantes. No decorrer das visitas os assistidos relatavam o quanto a interação com os cães influenciava positivamente na relação com os outros colegas hospitalizados e equipe de profissionais envolvidos. Nesse sentido, alguns estudos demonstram que pacientes com alterações psiquiátricas, principalmente depressão, quando participam das intervenções assistidas por animais tendem a reduzir o tempo de permanência no hospital e a tentativa de suicídio (MACHADO et al, 2008).

Constatou-se também que, os jogos lúdicos utilizados com a temática dos cães coterapeutas, facilitaram a adesão e participação dos pacientes durante as visitas. Pois, tais atividades proporcionam momentos de entretenimento e relaxamento, permitindo que os assistidos viessem a esquecer dos seus problemas cotidianos e até mesmo o motivo da internação (SILVEIRA, 2011).Confirmado pelo relato da terapeuta ocupacional, que ressalta uma melhora momentânea dos pacientes, facilitando a intervenção profissional durante e em momentos posteriores a atividade em função da diminuição do estresse, do desenvolvimento dos sentimentos de afeição, carinho e da facilidade da expressão de sentimentos potencializando a interação social entre os mesmos e os profissionais envolvidos. Considerando assim, que a utilização de cães durante as atividades, propicia estímulo, elevação da autoestima e a reintegração à sociedade, por meio do contato social que o animal permite (KOBAYASHI et al., 2009).

Neste contexto, deve-se considerar os assistidos, na sua singularidade, em diversas dimensões. De maneira, que o cuidado com a vida seja primordial. Por isso, é imprescindível que durante as intervenções assistidas por animais estejam envolvidos uma equipe multidisciplinar, para ter esse olhar para o paciente e todos os envolvidos. Dessa forma, estaremos tornando o ambiente hospitalar mais humanizado e nos tornando cada vez mais profissionais humanitários e capacitados. 


\section{CONSIDERAÇÕES FINAIS}

Conclui-se que, as intervenções assistidas por animais realizadas em pacientes hospitalares em ambas as instituições da cidade de Pelotas, foram realmente benéficas. Pois, foram constatados pelos profissionais envolvidos inúmeros benefícios, como a interação social, mudança de humor, melhora na motricidade e na qualidade de vida.

\section{REFERÊNCIAS}

ABRHÃO, F.; CARVALHO, M. C. Educação Assistida por Animais como recurso pedagógico na educação regular e especial:revisão bibliográfica. Revista Cientifica Digital da Faetec, Rio de Janeiro, v. 1, n.1, 2015.

CHELINI, M. O. M.; OTTA, E. Terapia Assistida por Animais. São Paulo: Manole, 2016.

GODOY, A. C.; DANZIN, S. S. Atividades Assistidas por Animais: aspectos revisivos sob um olhar pedagógico. 2007. Disponível em: <http://www.researchgate.net/ publication/266328629_Atividades_assistidas_por_animais_aspectos_revisivos_ sob_um_olhar_pedagogico,2007>. Acesso em: 17 fev. 2018.

ICHITANI, T. Efeito da Atividade Assistida por Animais na sensação de dor em crianças e adolescentes hospitalizados. (Dissertação de Mestrado). São Paulo: Pontifíca Universidade Católica de São Paulo, 2015.

KOBAYASHI, T.C. et al. Desenvolvimento e implantação de Terapia Assistida por Animais em hospital universitário. Revista Brasileira de Enfermagem, v. 4, n. 62,2009.

MACHADO, J. D. A. C.et al. Terapia assistida por animais (TAA). Revista Científica Eletrônica de Medicina Veterinária, São Paulo, v.6, n.10, p. 1-7, 2008.

MARCOLINO, J. A. M. et al. Escala hospitalar de ansiedade e depressão: estudo da validade de critérios e da confiabilidade com pacientes no pré-operatório. Revista Brasileira de Anestesiologia, São Paulo, v.57,n. 1, p. 52-62, 2007.

NOBRE,M. O.et al. Intervenções Assistidas por Animais: uma nova perspectiva na educação. Revista Eletrônica de Veterinária, Pelotas, v.18,n.2, p. 1-8, 2017.

RIBEIRO, V. P.et al. Interação lúdica na atividade assistida por cães em pediatria. Revista Enfermagem Foco, Pelotas, v.8, n. 1, p. 7-11, 2011. 
SILVA, D. M. G. V.et al. Qualidade de vida na perspectiva de pessoas com problemas respiratórios crônicos: a contribuição de um grupo de convivência. Revista Latino-Americana de Enfermagem, São Paulo, v.13, n.1, p. 7-14,2005.

SILVEIRA, I.R.; SANTOS, N.C.; LINHARES, D.R. Protocolo do programa de Assistência Auxiliada por Animais no hospital universitário. Revista da Escola de Enfermagem da USP, São Paulo, v.1, n.45, 2011.

VACCARI, A.M.H; ALMEIDA, F.A. A importância da visita de animais de estimação na recuperação de crianças hospitalizadas. [Trabalho de Conclusão de Curso]. São Paulo: Faculdade de Enfermagem do Hospital Israelita Albert Einstein, 2007.

YAMAMOTO, K.C.M. Avaliação fisiológica e comportamental de cães utilizados em Terapia Assistida por Animais (TAA). Revista Arquivo Brasileiro de Medicina Veterinária e Zootecnia, [Belo Horizonte], v.64,n.3, 2012.

LIMA, C. M

Imagem: Marcos Santos / USP Imagens

Data de recebimento: 04 de março de 2018.

Data de aceite para publicação: 26 de março de 2018. 